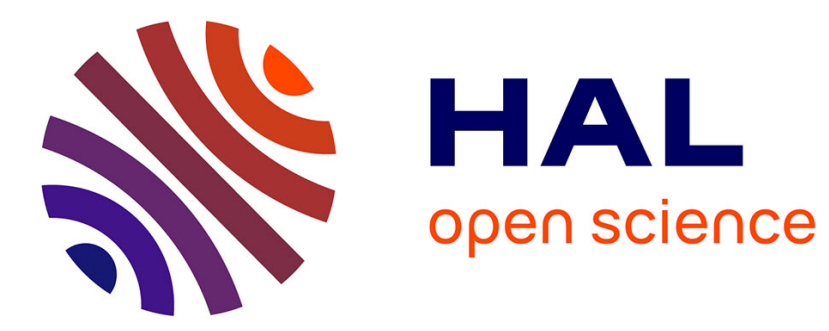

\title{
Comparative study and link between mesoscopic and energetic approaches in high cycle multiaxial fatigue
}

Franck Morel, Thierry Palin-Luc, Catherine Froustey

\section{To cite this version:}

Franck Morel, Thierry Palin-Luc, Catherine Froustey. Comparative study and link between mesoscopic and energetic approaches in high cycle multiaxial fatigue. International Journal of Fatigue, 2001, 23 (4), pp.317-327. 10.1016/S0142-1123(00)00100-6 . hal-01372757

\section{HAL Id: hal-01372757 https://hal.science/hal-01372757}

Submitted on 27 Sep 2016

HAL is a multi-disciplinary open access archive for the deposit and dissemination of scientific research documents, whether they are published or not. The documents may come from teaching and research institutions in France or abroad, or from public or private research centers.
L'archive ouverte pluridisciplinaire $\mathbf{H A L}$, est destinée au dépôt et à la diffusion de documents scientifiques de niveau recherche, publiés ou non, émanant des établissements d'enseignement et de recherche français ou étrangers, des laboratoires publics ou privés. 


\title{
Comparative study and link between mesoscopic and energetic approaches in high cycle multiaxial fatigue
}

\author{
Franck Morel ${ }^{\mathrm{a}, *}$, Thierry Palin-Luc ${ }^{\mathrm{b}}$, Catherine Froustey ${ }^{\mathrm{b}}$ \\ a Laboratoire de Mécanique et de Physique des Matériaux, UMR 6617, ENSMA, Site du Futuroscope, BP 109, 86960 Futuroscope Cedex, \\ France \\ b Laboraroire Matériaux Endommagement Fiabilité et Ingénierie des Procédés, EA 2727, ENSAM, Esplanade des Arts et Métiers, 33405 \\ Talence Cedex, France
}

\begin{abstract}
Multiaxial fatigue analysis can be categorized into several viewpoints, i.e. empirical formulae, methods based on stress invariants, critical plane approaches, models using averages of stress quantities and energetic considerations. The aim of this paper is not to survey the current state of knowledge concerning multiaxial fatigue but to critically examine two endurance criteria so as to prove that a direct link can be established between them.

The firs of the two methods, proposed by Papadopoulos, has been built by exploring the fatigue of metals from the mesoscopic scale, that is from the scale of the metal grains of a metallic aggregate. The localized plastic strains developping in some less favourably oriented crystals is considered to be the main cause of fatigue crack nucleation. According to relationships between macroscopic and mesoscopic quantities, this model is finall expressed in terms of the usual macroscopic stresses relative to an elementary material volume. The second approach proposed by Froustey and Lasserre is an energetic based criterion. It has been deduced from experimental observations concerning multiaxial endurance limit and states that crack initiation occurs as soon as the total strain energy density exceeds a critical value.

This paper shows that the critical value of the accumulated mesoscopic plastic strain used by Papadopoulos to characterize the endurance limit can be estimated with the global strain energy density at the macroscopic scale. Indeed, it is demonstrated that when dealing with in-phase or out-of-phase synchronous sinusoidal constant amplitude loadings, a single analytical formulation of these criteria can be written either with stress quantities or with energetic ones describing thus the same physical phenomenon. The mean stress influenc is discussed; the predictions of the two approaches are similar when the material remains quasi elastic. Another important result concerns the phase difference of the stress tensor components. Very few approaches are able to predict the independence of the fatigue strength on the phase difference between normal and shear stresses. The two proposed criteria reflec this phenomenon which has been experimentally observed for many metals subjected to combined bending-torsion loading. Nevertheless, this independence with regard to the phase shift is no more effective when dealing with some biaxial stress systems with two normal stresses. In this case the two models are consistent with the experimental results since they show a marked influence of the phase difference.
\end{abstract}

Keywords: High cycle fatigue; Multiaxial; Mesoscopic scale; Energy; Criterion; Metallic material

\section{Introduction}

The design of structures against high cycle multiaxial fatigue is a very important challenge for engineers. Many criteria have been proposed in the literature for

* Corresponding author. Tel.: +33-5-4949-8239; fax: +33-5-49498238.

E-mail address: morel@1mpm.ensma.fr (F. Morel). designers and several studies have been devoted to a critical assessment of them. The aim of this paper is not to survey the current state of knowledge concerning high cycle multiaxial fatigue but to critically examine two endurance criteria based on two very different approaches of the fatigue crack initiation prediction: critical plane and energy, so as to prove that a direct link can be established between them.

Different scales of material description can be used to study the fatigue strength of metals. Two scales will be 


\section{Nomenclature}

$a, b \quad$ material parameters of the mesoscopic approach

$\underline{n} \quad$ unit normal of a material plane

$t \quad$ time

A, B, C, D functions of the mesoscopic criterion

C macroscopic shear stress vector

E Young modulus

$\mathrm{H}, \mathrm{I}, \mathrm{J}, \mathrm{K}$ functions of the energy based criterion

$\mathrm{I}_{1, \mathrm{a}}, \mathrm{I}_{1, \mathrm{~m}}$ firs invariants of the alternating and mean parts of the stress tensor

$\mathrm{J}_{2, \mathrm{a}}, \mathrm{J}_{2, \mathrm{~m}}$ second invariants of the alternating and mean parts of the stress deviatoric tensor

$N(\varphi, \theta)$ normal stress on the material plane define by the angles $\varphi, \theta$

$T \quad$ loading period

T macroscopic resolved shear stress vector

$\bar{T}_{\mathrm{a}} \quad$ amplitude of the macroscopic resolved shear stress

$R_{\mathrm{m}} \quad$ maximum tensile strength

$\underline{\underline{S}}=S_{i j} \quad$ macroscopic stress deviatoric tensor

$\underline{\underline{E}}=E_{i j} \quad$ macroscopic strain tensor

$W \quad$ mean value on a period of the elastic strain energy density

$W d \quad$ distortion part of the elastic strain energy density mean value

$W s \quad$ spherical part of the elastic strain energy density mean value

$\beta \quad$ material parameter of the energy based approach

$\beta_{i j} \quad$ phase difference of the stress tensor components

$\gamma^{p} \quad$ mesoscopic plastic strain

$\varphi, \theta, \psi$ spherical co-ordinates of a unit normal vector for an easy elementary material slip plane

$\sigma_{\text {ten }_{-1}}^{\mathrm{D}} \quad$ endurance limit under fully reversed tension

$\sigma_{\text {bend }}^{\text {D }}{ }^{-1}$ endurance limit under fully reversed plane bending

$\sigma_{\text {rotbend }}$ endurance limit under fully reversed rotating bending $^{\mathrm{D}}$

$\tau_{\mathrm{to}_{-1}}^{\mathrm{D}} \quad$ endurance limit under fully reversed torsion

$v \quad$ Poisson ratio

$\omega$ pulsation

$\underline{\underline{\Sigma}}=\Sigma_{i j} \quad$ macroscopic stress tensor

$\Sigma_{\mathrm{H}, \max }, \Sigma_{\mathrm{H}, a}, \Sigma_{\mathrm{H}, m}$ maximum, amplitude and mean values of the hydrostatic stress

more particularly considered here: the macroscopic scale (i.e. the usual scale of engineer or the scale of the continuous medium) and the mesoscopic scale (i.e. the scale of the grains of a metallic aggregate). In high cycle fatigue where macroscopic plastic strains are negligible, fatigue theories can be distinguished according to these two scales. Many fatigue criteria [20-23] have been proposed within the macroscopic scale, i.e. the stress, strain or energetic quantities arising in the various criteria are those reached by engineers by means of measurement (strain gauges) or calculation (finit element methods). For instance, Froustey et al. [1] proposed an energy based criterion stating that crack initiation occurs as soon as the distortion strain energy density exceeds a critical value; this approach, described in this paper, is called the energy based criterion. However since fatigue is also a phenomenon taking place at the scale of the grains of the metal, some authors [2-7,24], following the initial work of Dang Van [2], used a mesoscopic scale theory that provides (according to a plasticity analysis) a precise description of damage accumulation in some less plastically resistant grains. An important contribution to this approach is due to Papadopoulos whose last criterion is described below.

Following the presentation of the two above mentioned criteria (Papadopoulos and Froustey et al.), some experimental data obtained with in- or out-of-phase synchronous sinusoidal constant amplitude combined loadings are compared with the predictions of the two approaches. Then, their analytical formulations are compared to look for a link between mesoscopic and macroscopic energy based approaches. The mean load effect and the phase influenc are discussed.

\section{A mesoscopic scale approach}

In high cycle fatigue, the macroscopic local behaviour of a mechanical component remains most of the time 
elastic even if local stress concentrations can induce local plastic strains. Indeed, near the fatigue limit, elastic shakedown is reached after a few cycles and consequently the following material response is purely elastic. However, at the mesoscopic scale of the order of the grain size (or some grain sizes), the metal consisting of crystals of random orientation is not isotropic nor homogeneous. Such structure induces a fluctuatio of stress and strain among these grains. It seems then natural to assume that in some less plastically resistant grains, the mesoscopic stress can locally exceed the yield limit. If the cyclic response of the plastically deformed grains is not elastic shakedown then it can be assumed that a crack is likely to occur. Indeed, despite that the mechanisms which may lead to a fatigue limit in HCF are most of the time described as the grain boundary blockage for cracks of length less than the order of the grain size, some authors [2-7] consider that the elastic shakedown of the cyclic microplasticity is another source of a fatigue limit (which is generally lower than the one related to grain boundary blockage of microcracks). In this framework, the estimation of the stress and strain field at the mesoscopic scale can be carried out, as Dang Van [2] did, according to the Lin Taylor model.

To build an endurance criterion by means of this model, Papadopoulos [4-6] assumed that the crystals of a metallic aggregate follow a combined isotropic and kinematical hardening rule when flowin plastically. It is long known that metal grains possess some preferred orientations (slip systems) along which plastic strain can develop (Fig. 1). A slip plane and a slip direction on this plane constitute each slip system. The author showed that the accumulated plastic strain along a slip direction on a slip plane induced by an external cyclic load becomes nearly proportional to the macroscopic resolved shear stress amplitude $T_{\mathrm{a}}$, when the number of load cycles increases indefinitely

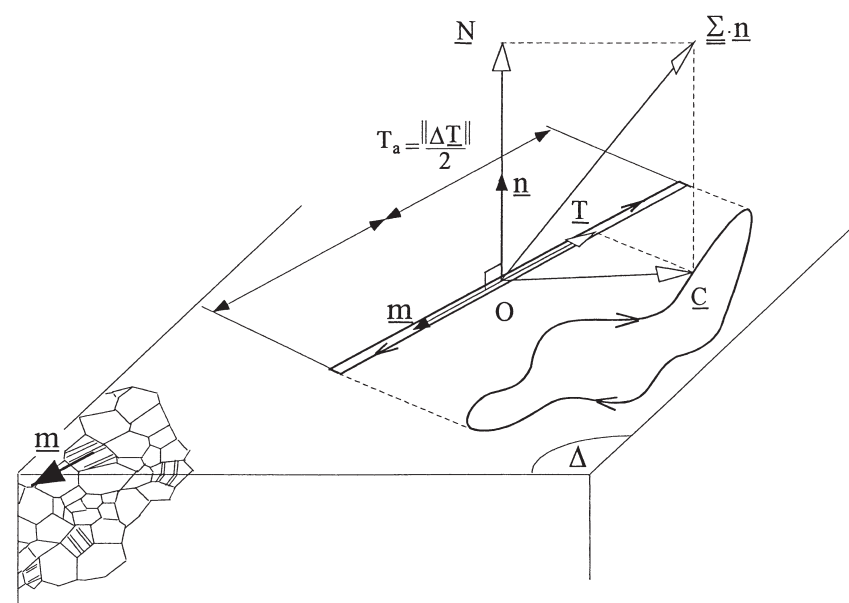

Fig. 1. Loading path of the shear stress vector $\underline{\mathbf{C}}$ and of the resolved shear stress vector $\underline{\mathbf{T}}$ on a material plane $\Delta$. $\sum_{\infty} \Delta \gamma^{p} \propto T_{\mathrm{a}}$

To avoid that the accumulated plastic strain exhausts the ductility of the crystal, a critical value can be define as a material parameter. The limitation of this mechanical quantity leads to a condition precluding the creation of a micro-crack within an elementary material volume. Although a so define fatigue criterion could be of interest to investigate the fatigue strength of single crystals, it is of no use within the engineering framework since in this context one has to prevent the creation of a fatigue crack of the same size as the elementary volume.

This fatigue engineering criterion is then based on two average measures. The firs one is related to the plastic strain accumulated in all the flowin crystals within the elementary volume:

$$
\sqrt{\left\langle T_{\mathrm{a}}^{2}\right\rangle}=\sqrt{5} \sqrt{\frac{1}{8 \pi^{2}} \int_{\varphi=0}^{2 \pi} \int_{\theta=0}^{\pi} \int_{\psi=0}^{2 \pi}\left(T_{\mathrm{a}}(\varphi, \theta, \psi)\right)^{2} \mathrm{~d} \psi \sin \theta \mathrm{d} \theta \mathrm{d} \varphi}
$$

The angle $\psi$ varying from 0 to $2 \pi$ covers all the gliding directions on a material plane whereas the angles $\varphi$ and $\theta$ varying from 0 to $2 \pi$ and from 0 to $\pi$, respectively, cover all the possible orientations of the material plane inside the elementary volume (Fig. 2).

But the limitation of this average measure cannot lead to a criterion able to encompass all the experimental fatigue knowledge. Indeed, it is now well established that the normal stress $N(\varphi, \theta)$ plays a key role on the
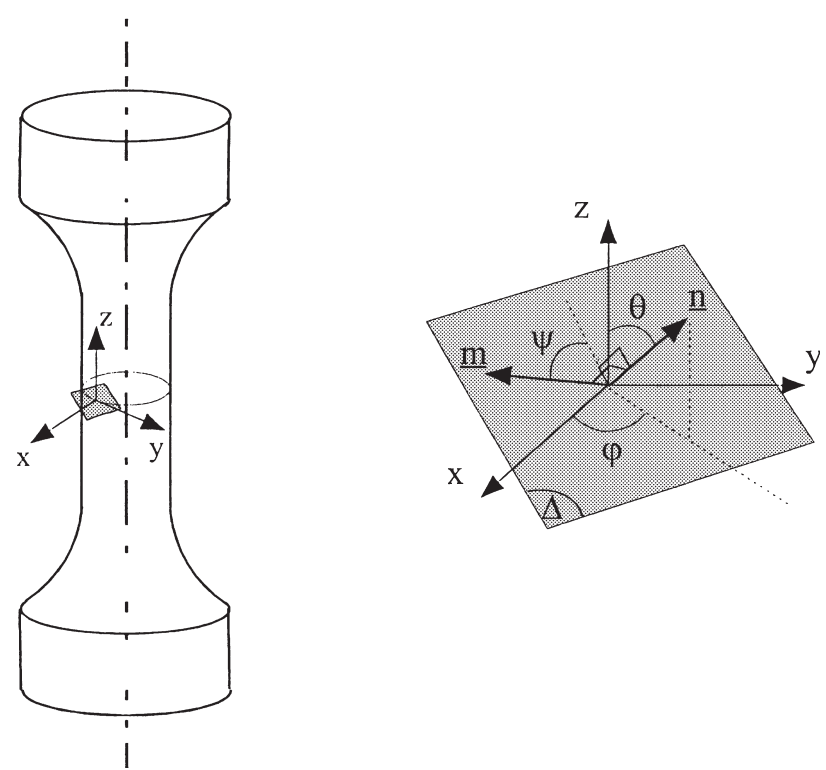

Fig. 2. Material plane $\Delta$ define by means of the spherical coordinates $\theta$ and $\varphi$ of the normal $\underline{\mathbf{n}}$ and direction $\underline{\mathbf{m}}$ on the plane located with the angle $\psi$ taken from an arbitrary but fixe direction on $\Delta$. 
fatigue strength. A second average measure is then introduced:

$$
\langle N\rangle=\frac{1}{4 \pi} \int_{\varphi=0 \theta=0}^{2 \pi} \int_{\theta}^{\pi} N(\varphi, \theta) \sin \theta \mathrm{d} \theta \mathrm{d} \varphi
$$

It is worth mentioning that this volumetric mean is nothing else but the hydrostatic stress $\Sigma_{\mathrm{H}}$.

Finally, the multiaxial endurance criterion is define as an inequality applied to a linear combination of the average quantities proposed above:

$\sqrt{\left\langle T_{\mathrm{a}}^{2}\right\rangle}+a \Sigma_{\mathrm{H}, \max } \leq b$

The two material parameters $a, b$ can be identifie from two fatigue limits, for example the fatigue limits in fully reversed tension-compression $\sigma_{\mathrm{ten}_{-1}}^{\mathrm{D}}$ and in fully reversed torsion $\tau_{\mathrm{to}_{-1}}^{\mathrm{D}}$ [Eq. (5)]. But the reference uniaxial fatigue limit has to be chosen according to the type of loading: $\sigma_{\text {ten }_{-1}}^{\mathrm{D}}$ for tension, $\sigma_{\text {bend }_{-1}}^{\mathrm{D}}$ for plane bending or $\sigma_{\text {rotbend }}^{\mathrm{D}}$ for rotative bending.

$a=3 \frac{\tau_{\mathrm{to}_{-1}}^{\mathrm{D}}}{\sigma_{\text {bend }_{-1}}^{\mathrm{D}}}-\sqrt{3}$ and $b=\tau_{\mathrm{to}_{-1}}^{\mathrm{D}}$

This criterion is not convenient for any kind of metals. Indeed to reflec the beneficia effect on the fatigue strength of a negative hydrostatic stress, the parameter a must be positive. This condition leads to a restriction of the criterion applicability. Only metals for which the ratio $\tau_{\mathrm{to}_{-1}}^{\mathrm{D}} / \sigma_{\mathrm{ten}_{-1}}^{\mathrm{D}}>1 / \sqrt{3}$ can be analysed through this approach. The ratio must not also be too close to one since the class of brittle metals is not considered in this theory. Papadopoulos proposed to limit his criterion to the materials verifying the condition $0.6 \leq \tau_{\mathrm{to}_{-1}}^{\mathrm{D}} / \sigma_{\mathrm{ten}_{-1}}^{\mathrm{D}} \leq$ 0.8 .

\section{An energy based approach}

Froustey et al. proposed in 1992 [1] an energy based macroscopic formula based on the accumulation of the strain energy density. The aim of this global criterion is not to predict the propagation direction of a fatigue crack but to predict multiaxial fatigue crack initiation.

The authors consider the mean value on a load period $T$ of the elastic strain energy density $W$ define by Eq. (6) whatever the point $\mathrm{M}$ in the component, where $\Sigma_{i j}$ and $E_{i j}^{\mathrm{e}}$ are respectively the tensor of stresses and the tensor of elastic strains at the considered point function of time.

$W=\frac{1}{T} \int_{0}^{T} \frac{1}{2} \Sigma_{i j}(t) E_{i j}^{\mathrm{e}}(t) \mathrm{d} t$

As proposed by Tsybanev [9], a complete loading cycle is considered in order to distinguish rotative bending and plane bending. In the high cycle fatigue regime the material remains elastic at the macroscopic scale [8], thus $W$ is also the total strain energy density mean value.

For any periodic loading, the tensor of stresses $\Sigma_{i j}(t)$ can be considered as the sum of two tensors: the alternating part $\Sigma_{i j, \mathrm{a}}(t)$ and the mean value $\Sigma_{i j, \mathrm{~m}}$. The same consideration can be made for the strain tensor: $E_{i j}(t)=E_{i j, \mathrm{a}}(t)+E_{i j, \mathrm{~m}}$.

For any periodic loading, Froustey et al. [1] writes the total strain energy density as the sum of an alternating part $W a$ (due to $\Sigma_{i j, \mathrm{a}}(t)$ and $\left.E_{i j, \mathrm{a}}(t)\right)$ and the static part $W m$ (due to $\Sigma_{i j, \mathrm{~m}}$ and $E_{i j, \mathrm{~m}}$ ): $W=W a+W m$, with:

$W a=\frac{1}{T} \int_{0}^{T} \frac{1}{2} \sum_{i j, \mathrm{a}}(t) E_{i j, \mathrm{a}}^{\mathrm{e}}(t) \mathrm{d} t$ and $W m=\frac{1}{2} \sum_{i j, \mathrm{~m}} E_{i j, \mathrm{~m}}^{\mathrm{e}}$

Since fatigue crack initiation is depending on the hydrostatic stress and the alternating shear stress, Froustey and Lasserre have chosen to consider separately the spherical part Wsa of the alternating strain energy density $W a$ and its deviatoric part (distortion energy) $W d a$ Eq. (8). In the same way, they have considered the spherical part Wsm and the deviatoric part $W d m$ of the static strain energy density $W m$ Eq. (9).

$$
\begin{aligned}
& W s a=\frac{1-2 v}{6 \cdot E} \cdot \frac{1}{T} \int_{0}^{T} \mathrm{I}_{1, \mathrm{a}}^{2}(t) \mathrm{d} t \text { and } W d a=\frac{1+v}{E} \cdot \frac{1}{T} \int_{0}^{T} \mathrm{~J}_{2, \mathrm{a}}(t) \mathrm{d} t \\
& W s m=\frac{1-2 v}{6 \cdot E} \cdot \mathrm{I}_{1, \mathrm{~m}}^{2} \text { and } W d m=\frac{1+v}{E} \cdot \mathrm{J}_{2, \mathrm{~m}}
\end{aligned}
$$

where

$$
\begin{aligned}
\sum_{i j, \mathrm{a}}(t) & =\frac{\sum_{k k, \mathrm{a}}(t)}{3} \cdot \delta_{i j}+S_{i j, \mathrm{a}}(t) \sum_{i j, \mathrm{~m}}=\frac{\sum_{k k, \mathrm{~m}}}{3} \cdot \delta_{i j}+S_{i j, \mathrm{~m}} \\
\mathrm{I}_{1, \mathrm{a}}(t) & =\sum_{k k, \mathrm{a}}(t) \mathrm{I}_{1, \mathrm{~m}}=\sum_{k k, \mathrm{~m}} \\
\mathrm{~J}_{2, \mathrm{a}}(t) & =\frac{1}{2} S_{i j, \mathrm{a}}(t) S_{i j, \mathrm{a}}(t) \mathrm{J}_{2, \mathrm{~m}}=\frac{1}{2} S_{i j, \mathrm{~m}} S_{i j, \mathrm{~m}}
\end{aligned}
$$

Several workers showed that the fatigue strength of metallic materials is depending on the triaxiality of stresses [10]. For Froustey and Lasserre, the triaxiality degree of stresses is define by reference to the work of De Leiris [11]. This is the ratio between the spherical part of $W$ and its totality, it is define for alternating stresses, $\mathrm{d} T a$, and for mean stresses, $\mathrm{d} T m$.

$\mathrm{d} T a=\frac{W s a}{W a} \quad \mathrm{~d} T m=\frac{W s m}{W m}$

The influenc of the triaxiality degree of stresses is described for a given mean load by a normalised function $F$ varying between 0 and 1 , 


$$
\begin{aligned}
& \frac{W a}{W d a_{\mathrm{eq}}}=F(\mathrm{~d} T a, \beta)=\frac{1}{1-\mathrm{d} T a} \cdot\left\{1-\frac{1}{\beta} \cdot \ln \left[1+\mathrm{d} T a \cdot\left(\mathrm{e}^{\beta}\right.\right.\right. \\
& -1)]\}
\end{aligned}
$$

where $W d a_{\text {eq }}$ is the equivalent distortion energy generated at the endurance limit by a loading of pure distortion, i.e. torsion $(\mathrm{d} T a=0) . \beta$ is a material dependent parameter characteristic of the material stress triaxiality sensibility.

On the same basis, Eq. (12) gives the relation between the static strain energy density $\mathrm{Wm}$ and the spherical part of the equivalent strain energy density $W s m_{\mathrm{eq}}$ that would be generated by a pure triaxial loading.

$\frac{W m}{W s m_{\mathrm{eq}}}=\frac{\beta}{1-\mathrm{e}^{-\beta}} \cdot F(\mathrm{~d} T m, \beta)$

Finally, by the use of Eqs. (11) and (12) the authors have proposed an energy based multiaxial endurance criterion as a linear relation Eq. (13) between the equivalent pure distorsion energy density and the equivalent pure triaxial strain energy density at the endurance limit (Fig. 3). $W d a_{\text {tors }}$ corresponds to the elastic strain energy density at the endurance limit in fully reversed torsion.

$W d a_{\mathrm{eq}} \leq W d a_{\mathrm{tors}} \cdot\left[1-\frac{W s m_{\mathrm{eq}}}{W s m_{\text {triax }}}\right]$

For a static triaxial loading, the spherical part of the elastic strain energy density $W s m_{\text {triax }}$ is identifie from the elastic strain energy density at $R_{\mathrm{m}}$ (maximal tensile strength) and the function $F\left(\mathrm{~d} T m_{\text {uniax }}, \beta\right)$. This can be written as Eq. (14).

$W s m_{\text {triax }}=\frac{R_{\mathrm{m}}^{2}}{2 E} \cdot \frac{1-\mathrm{e}^{-\beta}}{\beta} \cdot \frac{1}{F\left(\mathrm{~d} T m_{\text {uniax }}, \beta\right)}$

The $\beta$ parameter is determined from two fully reversed endurance limits on smooth specimen. $\sigma_{\text {rotbend }}^{\text {D }}$ is the

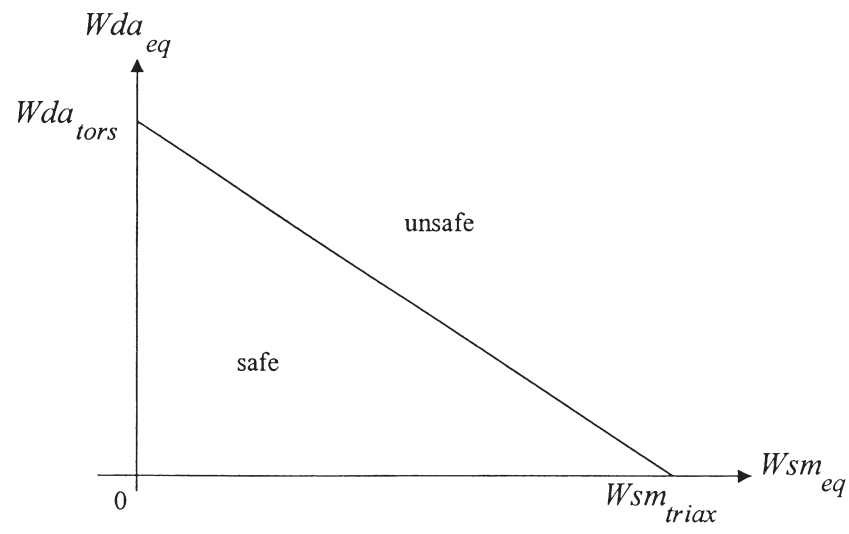

Fig. 3. Energy based criterion. endurance limit in rotative bending and $\tau_{\text {tors }_{-1}}^{\mathrm{D}}$ the endurance limit in torsion. Thus $\beta$ is solution of Eq. (15) derived from Eq. (11). For all the materials tested by Froustey et al. (cast irons, steels, aluminium alloys) this parameter is varying between 0 and 5 .

$$
\left(\frac{\sigma_{\text {rotbend }_{-1}}^{\mathrm{D}^{\mathrm{D}}}}{\tau_{\mathrm{to}_{-1}}^{\mathrm{D}}}\right)^{2}-3\left\{1-\frac{1}{\beta} \cdot \ln \left[1+\frac{1-2 v}{3}\left(\mathrm{e}^{\beta}-1\right)\right]\right\}=0
$$

\section{Link between the mesoscopic scale and the energy based approaches}

The two criteria described above can be applied to any kind of constant amplitude multiaxial loadings (sinus, triangle, square, ...) but only synchronous sinusoidal stress systems define by Eq. (16) will be examined in this part. All the usual multiaxial laboratory tests can then be considered (combined tension-torsion, biaxial tension, ....).

$\Sigma_{i j}(t)=\Sigma_{i j, \mathrm{a}} \sin \left(\omega t-\beta_{i j}\right)+\Sigma_{i j, \mathrm{~m}} i, j=x, y, z$

$\beta_{i j}$ is the phase difference between the (ij) stress component and a reference stress component (for example here $\Sigma_{x x}$ ). Let us notice that a sinusoidal loading is called synchronous if the frequency of all the stress components is the same.

\subsection{Criterion formulations}

\subsubsection{Mesoscopic scale approach}

The evaluation of the mechanical quantities $\sqrt{\left\langle T_{\mathrm{a}}^{2}\right\rangle}$ and $\Sigma_{\mathrm{H}, \max }$ is required to apply the mesoscopic scale criterion. The firs term comes out from an integration of the resolved shear stress amplitude acting along a slip direction on a slip plane. For loading as given by Eq. (16), the path described by the extremity of the shear stress vector on a material plane is an ellipse (Fig. 4). The corresponding major and minor semi-axes $C_{a}$ and $C_{b}$ are given by Eq. (17) where the quantities $A$, $\mathrm{B}, \mathrm{C}$ and $\mathrm{D}$ are functions of the phase difference $\beta_{i j}$ [7].

$\mathrm{C}_{\mathrm{a}, \mathrm{b}}=$

$$
\sqrt{\frac{\mathrm{A}^{2}+\mathrm{B}^{2}+\mathrm{C}^{2}+\mathrm{D}^{2}}{2} \pm \sqrt{\frac{\left(\mathrm{A}^{2}+\mathrm{B}^{2}+\mathrm{C}^{2}+\mathrm{D}^{2}\right)^{2}}{4}-(\mathrm{AD}-\mathrm{BC})^{2}}}
$$

As shown in [7] the amplitude $T_{\mathrm{a}}$ of the resolved shear stress acting along a slip direction $\mathrm{L}$ on the slip plane $\Delta$ here under consideration is equal to the half-length of the projection of the ellipse on the line L:

$$
T_{\mathrm{a}}=\sqrt{\mathrm{C}_{\mathrm{a}}^{2} \cos ^{2} \psi+\mathrm{C}_{\mathrm{b}}^{2} \sin ^{2} \psi}
$$




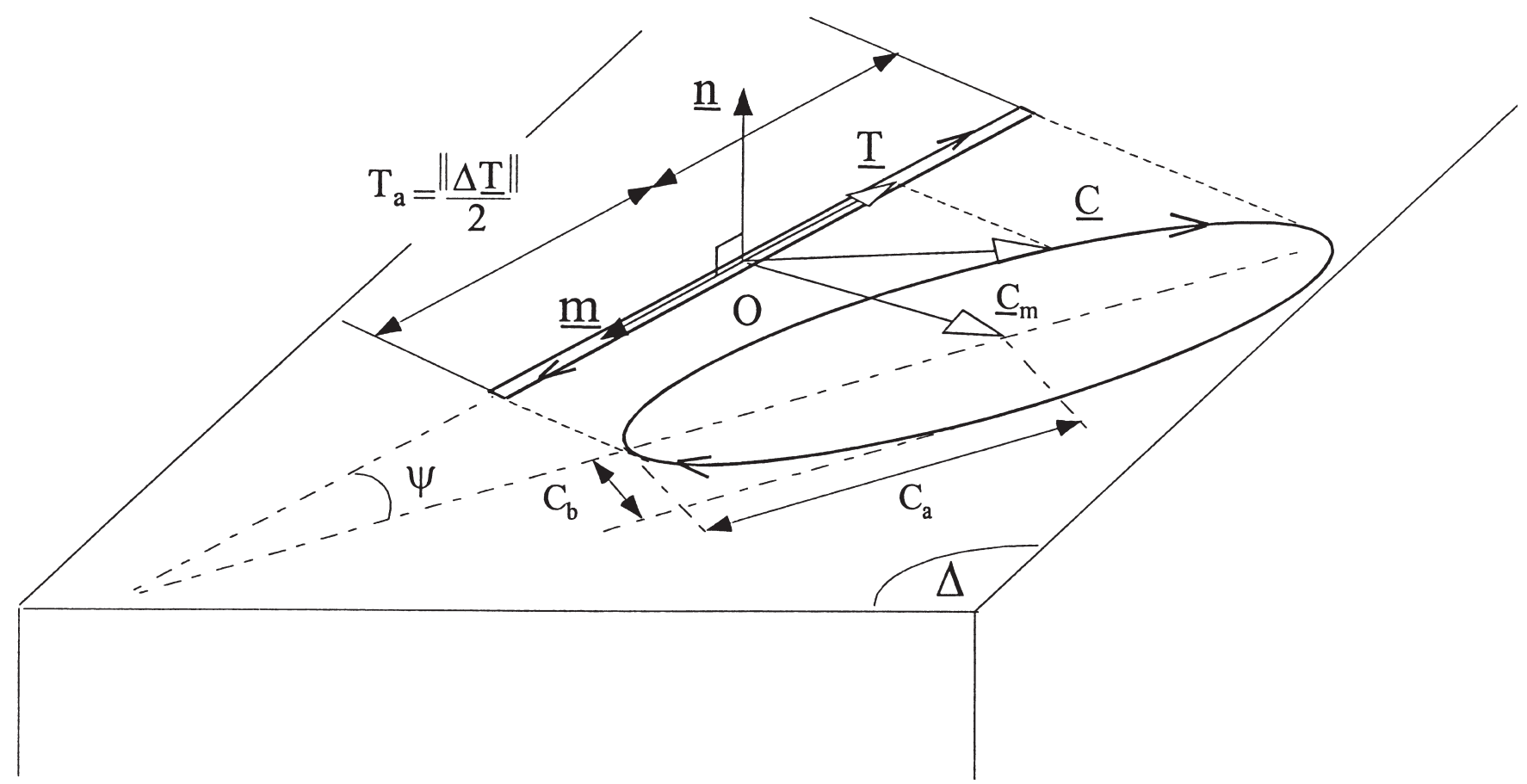

Fig. 4. Elliptic path on a plane $\Delta$ described by the shear stress vector extremity during a period of a synchronous sinusoidal out-of-phase bending and torsion (or tension and torsion) loading, from [7].

where $\psi$ is the angle formed between the direction of the major axis of the ellipse and the line L (Fig. 4).

Once the value of $T_{\mathrm{a}}$ has been introduced into Eq. (2) and after substantial analytical calculations, the average value achieves the simple following form:

$$
\begin{aligned}
& \sqrt{\left\langle T_{\mathrm{a}}^{2}\right\rangle}=\sqrt{\frac{1}{3}} \\
& {\left[\begin{array}{l}
\sum_{x x, \mathrm{a}}^{2}+\Sigma_{y y, \mathrm{a}}^{2}+\Sigma_{z z, \mathrm{a}}^{2}+3 \Sigma_{x y, \mathrm{a}}^{2}+3 \Sigma_{x z, \mathrm{a}}^{2}+3 \Sigma_{y z, \mathrm{a}}^{2}-\Sigma_{x x, \mathrm{a}} \Sigma_{y y, \mathrm{a}} \cos \left(\beta_{x x}-\beta_{y y}\right) \\
-\Sigma_{x x, \mathrm{a}} \Sigma_{z z, \mathrm{a}} \cos \left(\beta_{x x}-\beta_{z z}\right)-\Sigma_{y y, \mathrm{a}} \Sigma_{z z, \mathrm{a}} \cos \left(\beta_{y y}-\beta_{z z}\right)
\end{array}\right]^{1 / 2}}
\end{aligned}
$$

It is worth noting that this mean value is independent of the phase differences relative to the shear components of the stress tensor, i.e. $\beta_{x y}, \beta_{x z}, \beta_{y z}$.

For the maximum hydrostatic stress, one can reach the following analytical formulations:

$\Sigma_{\mathrm{H}, \max }=\Sigma_{\mathrm{H}, \mathrm{m}}+\Sigma_{\mathrm{H}, \mathrm{a}} \Rightarrow$

$\Sigma_{\mathrm{H}, \max }=\frac{1}{3}\left[\Sigma_{x x, \mathrm{~m}}+\Sigma_{y y, \mathrm{~m}}+\Sigma_{z z, \mathrm{~m}}\right]$

$+\frac{1}{3}\left[\begin{array}{l}\sum_{x x, \mathrm{a}}^{2}+\sum_{y y, \mathrm{a}}^{2}+\sum_{z z, \mathrm{a}}^{2}+2 \Sigma_{x x, \mathrm{a}} \Sigma_{y y, \mathrm{a}} \cos \left(\beta_{x x}-\beta_{y y}\right) \\ +2 \Sigma_{x x, \mathrm{a}} \Sigma_{z z, \mathrm{a}} \cos \left(\beta_{x x}-\beta_{z z}\right)+2 \Sigma_{y y, \mathrm{a}} \Sigma_{z z, \mathrm{a}} \cos \left(\beta_{y y}-\beta_{z z}\right)\end{array}\right]^{1 / 2}$

\subsubsection{Energy based approach}

If one consider the mean value over a loading period of the distortion elastic strain energy density due to the alternating part of the stress tensor $W d a$ (see Eq. (8)), it can be readily proved that for any out-of-phase synchronous sinusoidal loading $W d a$ is define by Eq. (21). The distortion elastic energy density corresponding to the mean (static) stresses $W d m$ is given by Eq. (22).

$$
\begin{aligned}
& W d a=\left(\frac{1+v}{6 E}\right)\left[\Sigma_{x x, \mathrm{a}}^{2}+\Sigma_{y y, \mathrm{a}}^{2}+\Sigma_{z z, \mathrm{a}}^{2}+3\left(\Sigma_{y z, \mathrm{a}}^{2}+\Sigma_{x z, \mathrm{a}}^{2}\right.\right. \\
& \left.+\Sigma_{x y, \mathrm{a}}^{2}\right)-\Sigma_{x x, \mathrm{a}} \Sigma_{y y, \mathrm{a}} \cos \left(\beta_{x x}-\beta_{y y}\right)-\Sigma_{x x, \mathrm{a}} \Sigma_{z z, \mathrm{a}} \cos \left(\beta_{x x}\right. \\
& \left.\left.-\beta_{z z}\right)-\Sigma_{y y, \mathrm{a}} \Sigma_{z z, \mathrm{a}} \cos \left(\beta_{y y}-\beta_{z z}\right)\right\rfloor \\
& W d m=\left(\frac{1+v}{3 E}\right)\left[\Sigma_{x x, \mathrm{~m}}^{2}+\Sigma_{y y, \mathrm{~m}}^{2}+\Sigma_{z z, \mathrm{~m}}^{2}-\Sigma_{x x, \mathrm{~m}} \Sigma_{y y, \mathrm{~m}}\right. \\
& \left.\quad-\Sigma_{y y, \mathrm{~m}} \Sigma_{z z, \mathrm{~m}}-\Sigma_{z z, \mathrm{~m}} \Sigma_{x x, \mathrm{~m}}+3\left(\Sigma_{x y, \mathrm{~m}}^{2}+\Sigma_{x z, \mathrm{~m}}^{2}+\Sigma_{y z, \mathrm{~m}}^{2}\right)\right]
\end{aligned}
$$

Moreover the spherical part of the strain energy density is given by:

$$
W S=\frac{1-2 v}{6 E}\left\{\frac{1}{T} \int_{0}^{T} \mathrm{I}_{1, \mathrm{a}}^{2}(t) \mathrm{d} t+\mathrm{I}_{1, \mathrm{~m}}^{2}\right\}
$$

where

$\mathrm{I}_{1, \mathrm{~m}}^{2}=\left(\Sigma_{x x, \mathrm{~m}}+\Sigma_{y y, \mathrm{~m}}+\Sigma_{z z, \mathrm{~m}}\right)^{2}$

and

$$
\begin{aligned}
& \mathrm{I}_{1, \mathrm{a}}^{2}(t)=\left[\Sigma_{x x, \mathrm{a}} \sin \left(\omega t-\beta_{x x}\right)+\Sigma_{y y, \mathrm{a}} \sin \left(\omega t-\beta_{y y}\right)\right. \\
& \left.\quad+\Sigma_{z z, \mathrm{a}} \sin \left(\omega t-\beta_{z z}\right)\right]^{2}
\end{aligned}
$$

After some calculations, the spherical parts of the static and alternating strain energy density, respectively $\mathrm{Wsm}$ and $W s a$, are: 


$$
\begin{aligned}
& W s m=\frac{1-2 v}{6 E} \mathrm{I}_{1, \mathrm{~m}}^{2}=\frac{1-2 v}{6 E}\left(\Sigma_{x x, \mathrm{~m}}+\Sigma_{y y, \mathrm{~m}}+\Sigma_{z z, \mathrm{~m}}\right)^{2} \\
& W s a=\frac{1-2 v}{6 E} \cdot \frac{1}{2}\left[\left(\Sigma_{x x, \mathrm{a}}^{2}+\Sigma_{y y, \mathrm{a}}^{2}+\Sigma_{z z, \mathrm{a}}^{2}\right)\right. \\
& +2 \Sigma_{x x, \mathrm{a}} \Sigma_{y y, \mathrm{a}} \cos \left(\beta_{x x}-\beta_{y y}\right)+2 \Sigma_{x x, \mathrm{a}} \Sigma_{z z, \mathrm{a}} \cos \left(\beta_{x x}-\beta_{z z}\right) \\
& \left.+2 \Sigma_{y y, \mathrm{a}} \Sigma_{z z, \mathrm{a}} \cos \left(\beta_{y y}-\beta_{z z}\right)\right\rfloor
\end{aligned}
$$

\subsection{Application of the two criteria to sinusoidal loadings}

The formulae giving the predictions of the two criteria for different sinusoidal loadings (usually used for fatigue tests) are shown in Table 1. All these predictions are illustrated in Fig. 5 for the 30NCD16 quenched and tempered steel whose characteristics are: $E=200 \mathrm{GPa}$, $\mathrm{v}=0.3, \mathrm{R}_{\mathrm{m}}=1200 \mathrm{MPa}, R p_{0.02}=895 \mathrm{MPa}, \sigma_{\mathrm{ten}_{-1}}^{\mathrm{D}}=560 \mathrm{MPa}$, $\sigma_{\text {rotbend }_{-1}}^{\mathrm{D}}=658 \mathrm{MPa}, \tau_{\mathrm{to}_{-1}}^{\mathrm{D}}=428 \mathrm{MPa}$. For the predictions shown in Fig. 5, the identificatio of the material parameters $a$ and $b$ of Papadopoulos criterion is carried out by means of two fully reversed endurance limits: torsion $\tau_{\mathrm{to}_{-1}}^{\mathrm{D}}$ and rotative bending $\sigma_{\text {rotbend }_{-1}}^{\mathrm{D}}$; according to the Papadopoulos classificatio this steel is hard because the ratio $\tau_{\mathrm{to}_{-1}}^{\mathrm{D}} / \sigma_{\mathrm{ten}_{-1}}^{\mathrm{D}}=0.76$ lies between 0.6 and 0.8 . The value of the $\beta$ parameter for this steel is 0.96 .

For biaxial stress state the predictions are shown in Fig. 6 for the 25CrMo4 steel [15] whose characteristics are: $R_{\mathrm{u}}=780 \mathrm{MPa}, R p_{0.02}=660 \mathrm{MPa}, \sigma_{\text {ten }_{-1}}^{\mathrm{D}}=361 \mathrm{MPa}$, $\tau_{\mathrm{to}_{-}}^{\mathrm{D}}=228 \mathrm{MPa}$. According to the Papadopoulos classificatio this material appears as a hard steel, its ratio $\tau_{\mathrm{to}_{-1}}^{\mathrm{D}} / \sigma_{\mathrm{ten}_{-1}}^{\mathrm{D}}=0.63$ being just at the low limit of the accept- able interval $[0.6,0.8]$. Since $\sigma_{\text {rotbend }-1}^{\text {D }}$ is unknown for these data, the $\beta$ parameter relative to the material stress triaxiality sensibility is identifie by means of $\sigma_{\mathrm{ten}_{-1}}^{\mathrm{D}}$. This leads to a $\beta$ value closed to zero.

One can notice that both criterion predictions are closed together especially if the material is not loaded over its static yield stress. They are sensitive to the phase differences $\beta_{i j}$ only for biaxial stress state with two normal stresses as shown in Fig. 6 . These points will be discussed later. For biaxial stress state it has to be noted that the reference uniaxial endurance limit used for the mesoscopic criterion is $\sigma_{\text {ten }_{-1}}^{\mathrm{D}}$.

\subsection{Set up of a direct link for synchronous sinusoidal loadings}

As $W d m$ represents the deviatoric part of the static strain energy density, it will not appear in the link between the two approaches since it is assumed in Papadopoulos theory that the mean part of the deviatoric tensor has no effect on fatigue limit.

Let us focus then on the other elastic strain energy components whose analytical expressions have been previously given.

First of all, concerning the average measure $\sqrt{\left\langle T_{\mathrm{a}}^{2}\right\rangle}$ of the macroscopic resolved shear stress amplitude $T_{\mathrm{a}}$ used in Papadopoulos criterion and given by Eq. (19), it appears from expression (21) definin the distortion elastic energy density $W d a$ due to the alternating part of the stress tensor that a simple relation relates these two quantities:

$W d a=\frac{1+v}{2 E}\left(\sqrt{\left\langle T_{\mathrm{a}}^{2}\right\rangle}\right)^{2}$

\begin{tabular}{|c|c|c|}
\hline Stress tensor components (non nul values) & Energy based criterion predictions & Mesoscopic criterion predictions \\
\hline \multicolumn{3}{|l|}{ Uniaxial stress states } \\
\hline$\Sigma_{i j}(t)=\Sigma_{x x, \mathrm{~m}}+\Sigma_{x x, \mathrm{a}} \sin \omega t$ & $\sum_{x x, \mathrm{a}}^{2} \quad+\frac{\sum_{x x, \mathrm{~m}}^{2}}{\leq 1}$ & $\sqrt{1}_{\Sigma} \quad a_{s}+r \quad<h$ \\
\hline \multicolumn{3}{|r|}{$\sum_{x x, \mathrm{a}}+\frac{-}{3}\left(\sum_{x x, \mathrm{a}}+\sum_{x x, \mathrm{~m}}\right) \leq b$} \\
\hline \multicolumn{3}{|l|}{ Pure torsion } \\
\hline$\Sigma_{i j}(t)=\Sigma_{x y, \mathrm{~m}}+\Sigma_{x y, \mathrm{a}} \sin \omega t$ & $\frac{\sum_{x y, \mathrm{a}}^{2}}{\left(\tau_{\mathrm{to}_{-1}}^{\mathrm{D}}\right)^{2}}+\frac{\sum_{x y, \mathrm{~m}}^{2}}{R_{\mathrm{m}}^{2}} \leq 1$ & $\Sigma_{x y, \mathrm{a}} \leq b$ \\
\hline & $\overline{2(1+v) \cdot F\left(\mathrm{~d} T m_{\text {uniax }}, \beta\right)}$ & \\
\hline \multicolumn{3}{|c|}{ Combined bending and torsion or combined tension and torsion } \\
\hline$\Sigma_{x x}(t)=\Sigma_{x x, \mathrm{~m}}+\Sigma_{x x, \mathrm{a}} \sin \left(\omega t-\beta_{x x}\right)$ & $\sum_{x x, \mathrm{a}}^{2} \quad \sum_{x x, \mathrm{~m}}^{2} \leq 1$ & $\sqrt{\sum_{x x, \mathrm{a}}^{2}+\Sigma^{2}}+a$ \\
\hline$\Sigma_{x y}(t)=\Sigma_{x y, \mathrm{~m}}+\Sigma_{x y, \mathrm{a}} \sin \left(\omega t-\beta_{x y}\right)$ & $\left(\sigma_{\text {rotbend }}{ }_{-1}^{2}\right)^{2} H^{2} R_{\mathrm{m}}^{2} I^{2}=1$ & $\sqrt{3}$ \\
\hline \multicolumn{3}{|c|}{ Tension-compression on thin wall tube with internal-external pressure } \\
\hline$\Sigma_{x x}(t)=\Sigma_{x x, \mathrm{~m}}+\Sigma_{x x, \mathrm{a}} \sin \left(\omega t-\beta_{x x}\right)$ & $\frac{\sum_{x x, \mathrm{a}}^{2}}{\left(\sigma_{\text {rotbend }_{-1}}^{\mathrm{D}}\right)^{2} \mathrm{~J}^{2}}+\frac{\sum_{x x, \mathrm{~m}}^{2}}{R_{\mathrm{m}}^{2} \mathrm{~K}^{2}} \leq 1$ & $\sqrt{\frac{1}{3}} \sqrt{\sum_{x x, \mathrm{a}}^{2}+\sum_{y y, \mathrm{a}}^{2}-\sum_{x x, \mathrm{a}} \Sigma_{y y, \mathrm{a}} \cos \left(\beta_{x x}-\beta_{y y}\right)}$ \\
\hline$\Sigma_{y y}(t)=\Sigma_{y y, \mathrm{~m}}+\Sigma_{y y, \mathrm{a}} \sin \left(\omega t-\beta_{y y}\right)$ & & $\left.i_{x x, \mathrm{~m}}+\sum_{y y, \mathrm{~m}}+\sqrt{\sum_{x x, \mathrm{a}}^{2}+\sum_{y y, \mathrm{a}}^{2}+2 \sum_{x x, \mathrm{a}} \Sigma_{y y, \mathrm{a}} \cos \left(\beta_{x x}-\beta_{y y}\right)}\right) \leq b$ \\
\hline
\end{tabular}

Table 1

Predictions of the energy based criterion and of the mesoscopic criterion for different stress states ${ }^{\mathrm{a}}$

\footnotetext{
${ }^{\text {a }} \mathrm{H}, \mathrm{I}, \mathrm{J}, \mathrm{K}$ are function of the $\Sigma_{i j}$ components and given in Appendix A.
} 

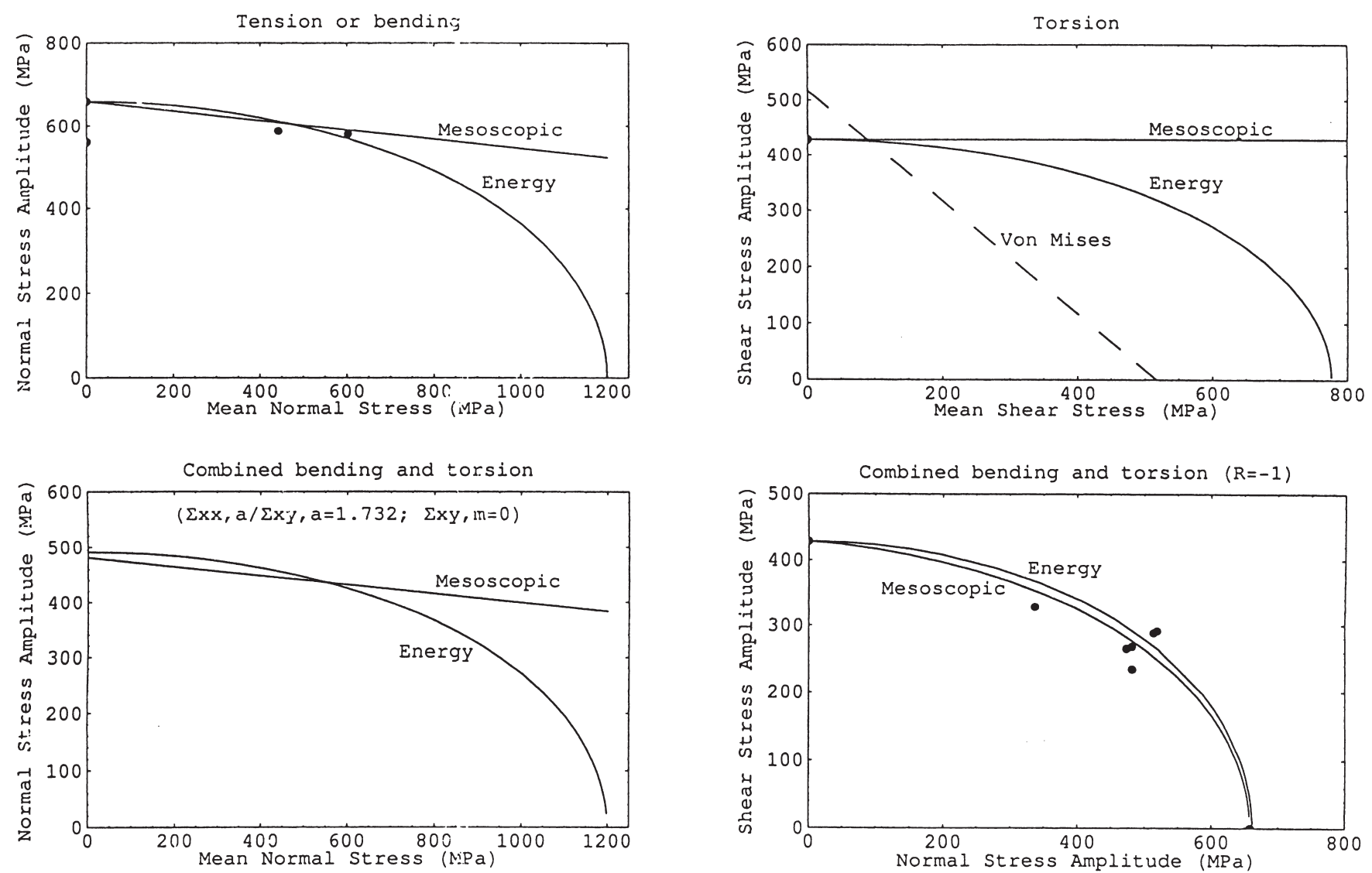

Fig. 5. Comparison of the two criterion predictions for different loadings on smooth specimens in 30NCD16 quenched and tempered steel (dark marks are experiments from Dubar [12]).

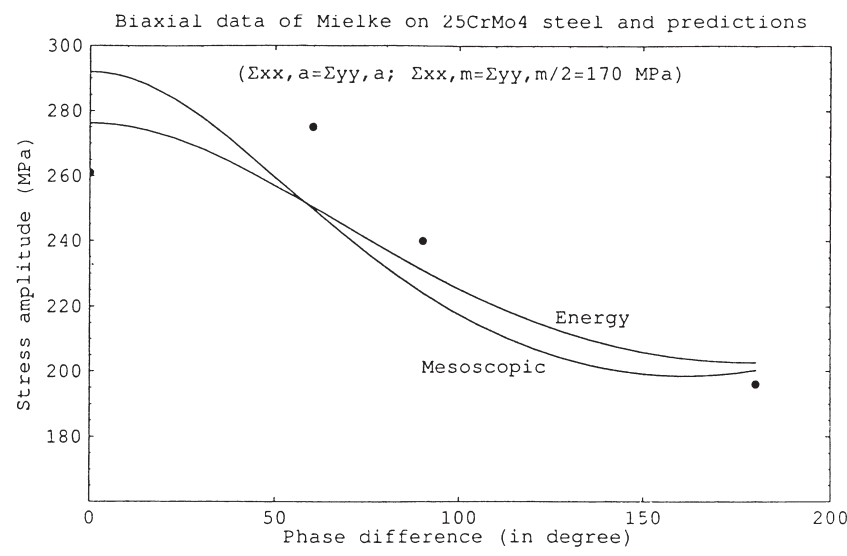

Fig. 6. Comparison of the two criterion predictions versus the phase difference in biaxial tension on smooth specimens in $25 \mathrm{CrMo} 4$ steel (dark marks are experiments from Mielke [15]).

The comparison of the two approaches can go on by studying the maximum value of the hydrostatic stress $\Sigma_{\mathrm{H} \text {,max }}$ and the spherical part of the elastic strain energy density. More precisely, from the relations (20), (26) and (27) one can readily show that:

$$
W s a=\frac{1-2 v 3}{2 E 2} \sum_{\mathrm{H}, \mathrm{a}}^{2}
$$

and

$$
W s m=\frac{1-2 v}{2 E} \sum_{\mathrm{H}, \mathrm{m}}^{2}
$$

Finally, from these relations (28), (29) and (30), the Papadopoulos criterion (4) can be expressed by means of the energetic parameters Wsa, Wsm and Wda:

$$
\begin{aligned}
& \sqrt{W d a \frac{2 E}{1+v}}+a \sqrt{\frac{2 E}{3(1-2 v)}}\left[\operatorname{sgn}\left(\mathrm{I}_{1, \mathrm{~m}}\right) \sqrt{W s m}\right. \\
& +\sqrt{2 W s a}] \leq b
\end{aligned}
$$

or in the same way:

$\sqrt{W d a}+a^{\prime}\left\lfloor\operatorname{sgn}\left(\mathrm{I}_{1, \mathrm{~m}}\right) \sqrt{W s m}+\sqrt{2 W s a}\right\rfloor \leq b^{\prime}$

where

$$
a^{\prime}=a \sqrt{\frac{1+v}{3(1-2 v)}} \text { and } b^{\prime}=\frac{b}{\sqrt{\frac{2 E}{1+v}}}
$$


with

$$
\operatorname{sgn}(x)=\left\{\begin{array}{l}
+1 \text { if } x>0 \\
0 \text { if } x=0 \\
-1 \text { if } x<0
\end{array}\right.
$$

\subsection{Discussion}

\subsubsection{Hypothesis agreement}

Eq. (28) proves that the plastic strain accumulated in all the flowin crystals within the elementary volume can be related to the alternating distortion energy mean value. In other words, an energetic integration carried out over a loading cycle leads to similar results than an integration of the resolved shear stress amplitude over each possible slip direction of every material plane passing through a point of a loading component. This correlation seems similar to the one made by Novhozilov [13] who already noticed (for monotonic loading) that the mean square of the shear stress over every material plane passing through a point of a loading component is equal to $2 / 5 J_{2}$ where $J_{2}$ is the second invariant of the deviatoric part of the stress tensor. To deal with endurance in fatigue, some authors (Simburger [14] and Grubisic [16]) proposed a criterion coming out from this relationship. However, some non-justifie assumptions lead to erroneous predictions [7]. For example, the influenc of a static shear stress on the torsion fatigue limit was predicted to be as strong as the effect of a static normal stress on the bending fatigue limit.

\subsubsection{Mean stress sensitivity}

Most of the tests in the high cycle fiel are carried out by applying loads with low mean values, that is, in condition where the material remains elastic at the macroscopic scale. There is no (or a few) hardening (or softening) mechanism activated. In such case, the predictions of the two criteria are close together and are always in good agreement with experimental data. However, if high mean stresses are applied, some differences between the predictions of the two formulations are observed.

When the mean value is a shear stress mean value, the mesoscopic approach does not predict any influenc of the mean load on the fatigue strength whereas the energetic based approach takes it into account. For high cycle fatigue (more than $10^{6}$ cycles) the independence of the fatigue limit in cyclic torsion with respect to superimposed mean torsion has been shown by Sines [17] who analysed 27 metals. Nevertheless this work has been used by Papadopoulos et al. [7] to confir that this mean stress independence is valid as far as yielding of the specimen is not reached, that is for $\tau_{\max }=\tau_{\mathrm{a}}+\tau_{\mathrm{m}}<\tau_{\mathrm{y}}$ where $\tau_{\mathrm{y}}$ is the yield limit value in torsion. The predictions of the energy based criterion are not inconsistent with this condition (Fig. 5). But the literature is too poor in high cycle fatigue data in torsion with a maximum shear stress significantl higher than the yield limit to critically discuss the accuracy of the two criterion predictions with high mean shear stress.

When the mean value is a normal mean value, the two models are built to reflec the dependence to static loads (for simple and combined loadings); this is in agreement with available experiments [7,17]. It is important to notice that when loads with high mean levels are imposed, an elastic shake down of the material must occur to reach fatigue limit (i.e. infinit life); the material tends then to recover a purely elastic response after a few cycles. Let us also mention another difference between the two criteria when the mean value is negative: mesoscopic approach shows an open safety area whereas the energetic based approach shows a close one.

\subsubsection{Phase shift sensitivity}

One common and striking feature of these two models is the phase difference influenc on criterion prediction for various stress states. As can be seen in the relations (19-22), (26) and (27) the mechanical quantities used in the criteria formulations are independent with respect to the phase difference $\beta_{x y}, \beta_{x z}$ and $\beta_{y z}$ related to the shear components of the stress tensor. It means that the two approaches predict no influenc of the phase shift on the fatigue strength when out-of-phase bending and torsion or out-of-phase push-pull and torsion loads are considered (see Table 1). Though this independence has been experimentally observed by many authors $[1,14,18]$, the widespread and popular fatigue criteria such as Crossland and Dang Van are unable to reflec this fact [19]. The proposals discussed in the paper are the few which deal properly with the phase effect on fatigue resistance for combined bending torsion loads. This common good predictive behaviour is all the more amazing because the two models have been built according to different approaches (i.e. mesoscopic and energetic).

The independence property with regard to the phase shift is only valid for combined bending (or tension) and torsion loads. For out-of-phase biaxial normal stress systems for example, the phase angles $\beta_{y y}$ appears in the criteria formulations (see Table 1). There are some experimental evidences of the marked influenc of the phase difference between two normal stresses. For instance, Mielke [15] showed on a $25 \mathrm{CrMo} 4$ steel and for a tension-compression and internal loading that the fatigue strength for the phase shift $\beta_{y y}=180^{\circ}$ is $33 \%$ lower than in the proportional conditions $\beta_{y y}=0^{\circ}$. These 
observations are clearly reflecte through the two models as shown in Fig. 6.

\section{Conclusions and prospects}

Two high-cycle fatigue criteria are presented and compared in this paper. They are based on two different approaches: the macroscopic criterion proposed by Froustey et al. is built according to energetic considerations while the second criterion proposed by Papadopoulos is based on a mesoscopic analysis of fatigue damage. Both models take into account proportional and non proportional multiaxial loadings. Moreover, in the case of out-of-phase, synchronous, sinusoidal stress systems simple analytical expressions are obtained. Thanks to these analytical formula, it has been proved that a direct link can be established between the energetic quantities of Froustey criterion and the average measure $\sqrt{\left\langle T_{\mathrm{a}}^{2}\right\rangle}$ of the macroscopic resolved shear stress amplitude $T_{\mathrm{a}}$ and the hydrostatic stress $\Sigma_{\mathrm{H} \text {,max }}$ of Papadopoulos work. This means that the upper-bound value of the accumulated plastic strain accumulated in all the flowin crystals within the elementary volume, $\sqrt{\left\langle T_{\mathrm{a}}^{2}\right\rangle}$, proposed by Papadopoulos is simply related to the distortion elastic energy density Wda due to the alternating stresses. One can then draw the conclusion that an energetic integration carried out over a loading cycle is similar to an integration of the resolved shear stress amplitude over each possible gliding directions of every material planes passing through a point of a loading component. This conclusion is closed to the one made by Novhozilov [13]: for monotonic loading the mean square of the shear stress over all material planes at a point is proportional to $\mathrm{J}_{2}$.

One striking feature of these two approaches is that they predict no influenc of the phase shift on the fatigue strength when out-of-phase bending and torsion or outof-phase push-pull and torsion loads are considered. This theoretical result is in good agreement with some experimental studies. Nonetheless this independence with regard to the phase shift is no more effective when dealing with biaxial stress systems. Indeed, the two models predict a marked influenc of the phase difference between two normal stresses. This strong dependence has been experimentally observed but still need to be confirmed

The two models present different dependence to the loading shape. For a proportional loading the average values $\sqrt{\left\langle T_{\mathrm{a}}^{2}\right\rangle}$ and $\Sigma_{\mathrm{H}, \max }$ of Papadopoulos criterion remain unchanged whatever the loading shape with time (sinusoidal, triangle, square) could be. The energy estimation requires though an integration with time which induces a dependence of this quantity over the loading shape. Since this influenc has not been widely studied in the scientifi literature about high cycle fatigue, the authors will draw no conclusion on this point. For

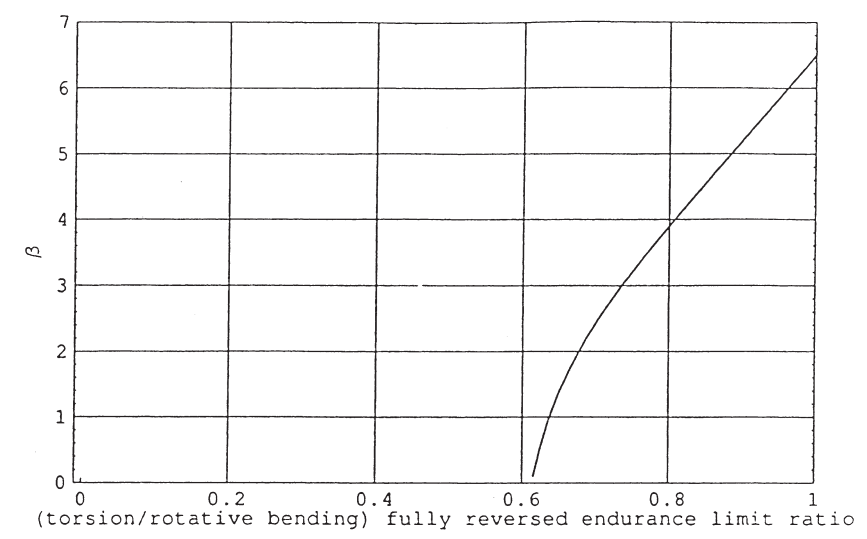

Fig. 7. Evolution of the $\beta$ parameter of the energy based criterion versus the ratio $\tau_{\mathrm{to}_{-1}}^{\mathrm{D}} / \sigma_{\text {rotbend }_{-1}}^{\mathrm{D}}$ for $v=0.3$.

instance, Dietmann et al. carried out fatigue tests on a ST35 steel in biaxial tension [25] with different wave forms but only under combined loadings and non-proportional loadings, not under simple loading such as push-pull. Experiments have to be done in the high cycle regime to investigate the loading shape influenc for simple and proportional loadings. For non proportional loadings the two criteria are loading shape dependent; this is in agreement with experiments in low cycle regime but there are not enough experimental data for long life to quantify the accuracy of the criteria.

Finally, let us point out too that the applicability of the Papadopoulos criterion is restricted to a class of material for which the inequality $\tau_{\mathrm{to}_{-1}}^{\mathrm{D}} / \sigma_{\mathrm{ten}_{-1}}^{\mathrm{D}}>1 / \sqrt{3}$ is observed. More precisely by considering that the model can not be applied to brittle materials, only 'hard metals' define by $0.6 \leq \tau_{\mathrm{to}_{-1}}^{\mathrm{D}} / \sigma_{\mathrm{ten}_{-1}}^{\mathrm{D}} \leq 0.8$ are concerned by this approach. The empirical Papadopoulos' classificatio according to the value of the ratio $\tau_{\mathrm{to}_{-1}}^{\mathrm{D}} / \sigma_{\mathrm{ten}_{-1}}^{\mathrm{D}}$ is linked with the $\beta$ parameter of the energy based approach. Indeed, $\beta$ is the solution of Eq. (16) depending on the ratio $\tau_{\mathrm{to}_{-1}}^{\mathrm{D}} / \sigma_{\text {rotbend }_{-1}}^{\mathrm{D}}$. Fig. 7 illustrates the evolution of $\beta$ versus this ratio which is not very different from the Papadopoulos ratio for metallic materials. This figur shows that $\beta$ is varying between 0 and 4 when $\tau_{\mathrm{to}_{-1}}^{\mathrm{D}} / \sigma_{\text {rotbend }_{-1}}^{\mathrm{D}}$ is varying between 0.6 and 0.8 . To deal with 'mild steels', $0.5 \leq \tau_{\mathrm{to}_{-1}}^{\mathrm{D}} / \sigma_{\mathrm{ten}_{-1}}^{\mathrm{D}} \leq 0.6$, Papadopoulos [4] already proposed a critical plane type criterion still based on the mesoscopic approach. In a future study, the authors will show that this last criterion can also be related to energetic quantities estimated from a material plane.

\section{Appendix A}

The different variables $\mathrm{H}, \mathrm{I}, \mathrm{J}, \mathrm{K}$ used to simplify the presentation of the equations in Table 1 are: 
$\mathrm{H}^{2}=\frac{F(\mathrm{~d} T a, \beta) / F\left(\mathrm{~d} T a_{\text {uniax }}, \beta\right)}{\left(1+\frac{2(1+v)}{\left(\Sigma_{x x, \mathrm{a}} / \Sigma_{x y, \mathrm{a}}\right)^{2}}\right)} \mathrm{J}^{2}=\frac{F(\mathrm{~d} T a, \beta) / F\left(\mathrm{~d} T a_{\text {uniax }}, \beta\right)}{1+\left(\frac{\Sigma_{y y, \mathrm{a}}}{\Sigma_{x x, \mathrm{a}}}\right)^{2}-2 \cdot v \cdot\left(\frac{\Sigma_{y y, \mathrm{a}}}{\Sigma_{x x, \mathrm{a}}}\right) \cdot \cos \left(\beta_{x x}-\beta_{y y}\right)}$

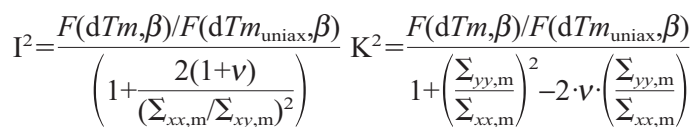

with $\mathrm{d} T a_{\text {uniax }}=d T m_{\text {uniax }}=\frac{1-2 v}{3}$ and

- in combined bending and torsion:

$\mathrm{d} T a=\frac{(1-2 v)\left(\Sigma_{x x, \mathrm{a}} / \Sigma_{x y, \mathrm{a}}\right)^{2}}{3\left(\Sigma_{x x, \mathrm{a}} / \Sigma_{x y, \mathrm{a}}\right)^{2}+6(1+v)}$

$\mathrm{d} T m=\frac{(1-2 v)\left(\Sigma_{x x, \mathrm{~m}} / \Sigma_{x y, \mathrm{~m}}\right)^{2}}{3\left(\Sigma_{x x, \mathrm{~m}} / \Sigma_{x y, \mathrm{~m}}\right)^{2}+6(1+v)}$

— in tension compression on thin wall tube with internal external pressure:

$\mathrm{d} T a=\frac{(1-2 v)}{3} \cdot \frac{1+\left(\Sigma_{x x, \mathrm{a}} / \Sigma_{y y, \mathrm{a}}\right)^{2}+2\left(\Sigma_{x x, \mathrm{a}} / \Sigma_{y y, \mathrm{a}}\right) \cos \left(\beta_{x x}-\beta_{y y}\right)}{1+\left(\Sigma_{x x, \mathrm{a}} / \Sigma_{y y, \mathrm{a}}\right)^{2}-2 v\left(\Sigma_{x x, \mathrm{a}} / \Sigma_{y y, \mathrm{a}}\right) \cos \left(\beta_{x x}-\beta_{y y}\right)}$

$\mathrm{d} T m=\frac{(1-2 v)}{3} \cdot \frac{1+\left(\Sigma_{x x, \mathrm{~m}} / \Sigma_{y y, \mathrm{~m}}\right)^{2}+2\left(\Sigma_{x x, \mathrm{~m}} / \Sigma_{y y, \mathrm{~m}}\right)}{1+\left(\Sigma_{x x, \mathrm{~m}} / \Sigma_{y y, \mathrm{~m}}\right)^{2}-2 v\left(\Sigma_{x x, \mathrm{~m}} / \Sigma_{y y, \mathrm{~m}}\right)}$

\section{References}

[1] Froustey C, Lasserre S, Dubar L. Validité des critères de fatigue multiaxiale à l'endurance en flexion-torsion Mat-Tech '92, IITT-International, France, 1992:79-85.

[2] Van Dang K. In: McDowell DL, Ellis R, editors. Advances in multiaxial fatigue, ASTM STP 1191. Philadelphia: ASTM, 1993:120-30

[3] Morel F. A fatigue life prediction method based on a mesoscopic approach in constant amplitude multiaxial loading. Fat Fract Engng Mat Struct 1998;21:241-56.

[4] Papadopoulos IV. Fatigue limit of metals under multiaxial stress conditions: the microscopic approach. Technical note no. I.93.101, Commission of the European Communities, Joint Research Centre, Ispra, Italy, ISEI/IE 2495/93, 1993.

[5] Papadopoulos IV. A new criterion of fatigue strength for out-of phase bending and torsion of hard metals. Int $\mathrm{J}$ Fat 1994;16(6):377-84

[6] Papadopoulos IV. A high cycle fatigue criterion applied in biaxial and triaxial out-of-phase stress conditions. Fat Fract Engng Mater Struct 1995;18(1):79-91.
[7] Papadopoulos IV, Davoli P, Gorla C, Filippini M, Bernasconi A. A comparative study of multiaxial high-cycle fatigue criteria for metals. Int J Fat 1997;19(3):219-35.

[8] Lemaitre J, Chaboche J-L. Mécanique des matériaux solides. Paris: Bordas, 1988.

[9] Tsybanev GV. An energy approach to fatigue tests and crack initiation stage determination. In: Problemy prochnosti. New York: Plenum Publishing Corp, 1994;2:12-27.

[10] Galtier A, Séguret J. Critères multiaxiaux en fatigue: exploitation en bureau d'études. Proposition d'un nouveau critère. Rev Franç. Méc 1990;1990-4:291-9.

[11] De Leiris H. Triaxialité des contraintes et critère de non fragilité. Bull l'Assoc Tech Marit Aéro 1969:481-91.

[12] Dubar L. Fatigue multiaxiale des aciers. Passage de l'endurance à l'endurance limitée, prise en compte des accidents géométriques. PhD thesis, no. 1992-19, ENSAM CER de Bordeaux, France, 1992.

[13] Novhozilov VV. Theory of elasticity. London: Pergamon Press, 1961.

[14] Simbürger A. Festigkeitsverhalten zäher Werkstoffe bei einer mehrachsigen, phasenverschobenen Schwingbeanspruchung mit körperfesten und veränderlichen Hauptspannungsrichtungen. Bericht, LBF, Nr. FB-121, Darmstadt, Germany, 1975.

[15] Mielke S. Festigkeitsverhalten metallischer Werkstoffe unter zweiachsig Schwingender Beanspruchung mit verschiedenen Spannungszeitverläufen. Diss., TH Aachen, Germany, 1980.

[16] Grubisic V. Ermüdungsfestigkeit unter Mehrachsiger Beanspruchung. Bericht, LBF, Darmstadt, Germany 1979:79-92.

[17] Sines G. Behaviour of metals under complex static and alternating stresses. In: Sines G, Waisman JL, Dolan TJ, editors. Metal fatigue. New York: McGraw-Hill, 1959:145-69.

[18] Froustey C, Lasserre S. Multiaxial fatigue endurance of 30NCD16 steel. Int J Fat 1989;11(3):169-75.

[19] Lasserre S, Froustey C. Multiaxial fatigue of steel - testing out of phase and in blocks: validity and applicability of some criteria. Int J Fat 1992;14(2):113-20.

[20] Socie D. Critical plane approaches for multiaxial fatigue damage assessment. In: McDowell DL, Ellis R, editors. Advances in multiaxial fatigue, ASTM STP 1191. Philadelphia: ASTM, 1993:7-36.

[21] McDiarmid DL. A general criterion for high cycle multiaxial fatigue failure. Fat Fract Engng Mat Struct 1991;14(4):429-53.

[22] Ellyin F, Golos K. Multiaxial fatigue damage criterion. J Engng Mat Technol Trans ASME 1988;110:63-8.

[23] Glinka G, Shen G, Plumtree A. A multiaxial fatigue strain energy density parameter related to the critical fracture plane. Fat Fract Engng Mat Struct 1995;18(1):37-46.

[24] Deperrois A. Sur le calcul des limites d'endurance des aciers, $\mathrm{Ph}$ D. Ecole Polytechnique, Paris, France, 1991.

[25] Dietmann H, Bhongbhibhat $T$, Schmid A. Multiaxial fatigue behaviour of steels under in-phase and out-of-phase loading, including different wave forms and frequencies. In: Kussmaul D, McDiarmid DL, Socie D, editors. Fatigue under biaxial and multiaxial loading. London: MEP, 1991:449-64. 